PTH-122 HOME PARENTERAL NUTRITION IN PALLIATIVE CARE: A RETROSPECTIVE STUDY TO IDENTIFY POTENTIAL PROGNOSTIC INDICATORS

B Drinkwater*, B Clarke, S Cooper. Russell's Hall Hospital, Dudley, UK

\subsection{6/gutjnl-2014-307263.568}

Introduction The use of home parenteral nutrition (HPN) in the palliative setting is less common in the UK than in Europe and the USA. It remains controversial with little evidence base supporting the existing guidance. Patient selection remains challenging. We aimed to identify potential prognostic indicators in palliative care patients receiving HPN using a retrospective analysis.

Methods Palliative care patients commenced on HPN were identified. Medical notes, computer records and the HPN database were accessed to identify patient demographics, primary diagnosis and aetiology of intestinal failure, blood test results potentially associated with prognosis (eGFR, albumin (not employed as a marker of malnutrition), CRP, $\mathrm{Hb}$ ), presence of ascites prior to starting HPN, and total number of nights on HPN. By dichotomising the blood results into CKD stage 3 or worse (eGFR $<60$ ), albumin $\leq 30, \mathrm{CRP}>50, \mathrm{Hb}<10$, we were able to derive Kaplan-Meier survival plots to identify statistical significance associated with survival.

Results From the HPN database of 111 patients, 20 (18\%) were identified as palliative. Six were male (30\%), and the median age (interquartile range (IQR)) was 56.4 (51.5-66.8) years; range 25.2-81.6. Between 2000 and 2006 four patients commenced palliative HPN, while 2007-2013 there were 16. At the time of data collection $80 \%(16 / 20)$ had died. The median number (IQR) of nights spent on HPN was 85 (19-352). The most common indication for HPN (aetiology of intestinal failure) was gastro-intestinal obstruction $(\mathrm{n}=13,65 \%)$ and short bowel syndrome following palliative surgical resection $(\mathrm{n}=4,20 \%)$.

The median (IQR) eGFR was 90 (53.5-90), with eGFR $<60$ $\mathrm{n}=4$, median (IQR) albumin 30 (24.5-35); low albumin $\leq 30$, $\mathrm{n}=10$; median $\mathrm{Hb} 10.4$ (9.3-10.9); $\mathrm{Hb}<10 \mathrm{n}=7$; median (IQR) CRP 10.5 (3.8-89.3), high CRP $>50 \mathrm{n}=4$. Of the 14 available case notes reviewed, there was evidence of ascites prior to starting HPN in $35.7 \%(5 / 14)$.

Kaplan-Meier survival plots identified a statistically significant shorter prognosis on HPN if the presenting albumin was $\leq 30 \mathrm{p}$ $=0.016$, median $66(5-115)$ days, and similarly if the CRP $>50$ $\mathrm{p}=0.04$, median (IQR) 98 (56-122). No significance was found with low $\mathrm{Hb}$, CKD stage 3 or worse or presence of ascites when compared with prognosis by Kaplan-Meier survival plots.

Conclusion We conclude that a low albumin (not a marker of malnutrition) and high CRP may help to predict those who are likely to survive less long on palliative HPN. When examining median days this appears most pertinent with a low albumin. There does not appear to be any significant association between 'presence of ascites', a low $\mathrm{Hb}$ or a low eGFR and survival time on HPN. While this study does not examine quality of life, the use of albumin and CRP may help direct whether HPN may be of benefit in the palliative setting.

Disclosure of Interest None Declared.

\section{PTH-123 QUALITY OF LIFE AND PERFORMANCE STATUS SCORES FOLLOWING INTESTINAL TRANSPLANTATION ARE SIMILAR TO THOSE OF PATIENTS ON HOME PARENTERAL NUTRITION IN THE UK}

${ }^{1} \mathrm{C}$ Pither*, 'S Duncan, ${ }^{2} \mathrm{~A}$ Butler, ${ }^{3} \mathrm{M}$ Stroud, ${ }^{3} \mathrm{~T}$ Smith, ${ }^{4} \mathrm{P}$ Calder, ${ }^{1} \mathrm{G}$ Rui, ${ }^{1} \mathrm{~B}$ Chukualim, 1) Woodward, 'J Green, ${ }^{1} \mathrm{R}$ Charlotte, ${ }^{1} \mathrm{~L}$ Sharkey, ${ }^{5} \mathrm{~S}$ Gabe, ${ }^{1} \mathrm{~S}$ Middleton. ${ }^{1}$ Gastroenterology, Cambridge University Hospitals NHS Foundation Trust, Cambridge, UK; ${ }^{2}$ Transplantation Surgery, Cambridge University Hospitals NHS Foundation Trust, Cambridge, UK; ${ }^{3}$ Gastroenterology, University Hospitals Southampton NHS Foundation Trust, Southampton, UK; ${ }^{4}$ Faculty of Medicine, University of Southampton, Southampton, UK; ${ }^{5}$ Gastroenterology, St Marks Hospital, London, UK

\subsection{6/gutjnl-2014-307263.569}

Introduction Survival following Intestinal transplantation (ITX) has improved, approaching that of home parenteral nutrition (HPN) at 5 years. We describe quality of life (QOL) and performance status (PS) on uncomplicated HPN, those with criteria for ITX due to complicated HPN (CHPN) and those after ITX.

Methods SF36 was used to assess QOL. Performance status was assessed by health related visual analogue scale (VAS), ECOG and Karnofsky performance score (KS).

Results [Table - grouped data]. There was a trend for QOL and PS to be lower on CHPN than HPN and ITX (not significant, NS)

Paired data for SF36 pretransplant (CHPN) and post-transplant revealed "general health "was the only function to significantly improve $(\mathrm{p}<0.05)$. Individual patients' total scores improved in $4 / 11$ with a statistically significant improvement in a further in $3 / 11(p<0.05)$ and were worse in $3 / 11$. PS showed a trend for improvement post ITX compared to CHPN and HPN [All Wilcoxon signed rank, NS].

Conclusion SF36 improves significantly in approximately 25\%, but falls in 25\% after ITX compared CHPN and trends suggest performance improvement after ITX. With more data effects of ITX on QOL and PS will emerge and if improvements are confirmed earlier transplantation for those dependent on HPN may be justifiable.

Disclosure of Interest None Declared.

\section{PTH-124 CHANGES IN THE INDICATIONS FOR REFERRAL OF ADULTS FOR INTESTINAL AND MULTIVISCERAL TRANSPLANTATION}

${ }^{1} \mathrm{C}$ Rutter* ${ }^{1} \mathrm{~L}$ Sharkey, ${ }^{2} \mathrm{~A}$ Butler, ${ }^{2} \mathrm{~N}$ Russell, ${ }^{3} \mathrm{~S}$ Gabe, ${ }^{2} \mathrm{~N}$ Jamieson, ${ }^{1} \mathrm{~J}$ Woodward, 'S Duncan, 'D Bond, 'I Green, 'S Middleton. 'Gastroenterology, Addenbrooke's Hospital, Cambridge, UK; ${ }^{2}$ Transplant Surgery, Addenbrooke's Hospital, Cambridge, UK; ${ }^{3}$ LennardJones Intestinal Failure Unit, St Mark's Hospital, London, UK

10.1136/gutjnl-2014-307263.570

\begin{tabular}{lllllll}
\multicolumn{1}{l}{ Abstract PTH-123 Table $\mathbf{1}$} \\
\hline
\end{tabular}

